\title{
Microsatellite Genotyping of Post-PCR Fluorescently Labeled Markers
}

BioTechniques 29:868-872 (October 2000)

\author{
H. Kondo, T. Tahira, H. \\ Hayashi $^{1}$, K. Oshima ${ }^{1}$ and \\ K. Hayashi \\ Kyushu University and 'Fukuo- \\ ka University School of Medi- \\ cine, Fukuoka, Japan
}

\section{INTRODUCTION}

Microsatellite markers are routinely used to construct genetic maps and to locate disease genes by linkage analysis. To perform microsatellite genotyping, the accuracy of fragment size identification is crucial. A fluorescence-based genotyping system using a sequence analyzer has been widely adopted because it offers both precise estimation of fragment size and analysis of data by a semi-automated, highthroughput system that includes allele identification software $(11,13)$.

Microsatellite markers are most commonly amplified with fluorescently labeled primers. This makes the whole procedure costly because synthesis of such primers is expensive compared with that of unlabeled primers. Another drawback arises with the use of fluorescently labeled primers: DNA polymerases such as Taq DNA polymerase tend to add a non-template nucleotide to the $3^{\prime}$ end of the PCR product, and the incomplete addition causes problems in allele identification for genotyping (1). To solve this problem, several modifications of the procedure are proposed: (i) specific PCR conditions are established for each pair of primers; (ii) the $5^{\prime}$ ends of primers are altered to contain a specific nucleotide that may either prohibit or promote nucleotide addition (9); (iii) DNA polymerase with exonuclease activity, such as $P f u$ polymerase, is used (12); or (iv) T4 polymerase is used after PCR to remove the added nucleotide (3). In spite of such measures, the persistent, incomplete addition or excess removal of the nucleotide sometimes hinders accu- rate size identification (3).

An alternative method for labeling PCR products involves the use of labeled dNTPs, which are internally incorporated during the amplification reaction. Although cost effective, this method is not suitable for precise estimation of the length of PCR products because the amplified fragments are chemically heterogeneous, which is caused by variability in the number of incorporated fluorescent nucleotides (5). Recently, we have developed a postPCR fluorescence-labeling method (5, 6). In this technique, PCR is performed with unlabeled primers, the $5^{\prime}$ ends of which are modified to contain either ATT or GTT, so that the $3^{\prime}$ ends of the complementary strands of the PCR products are either $\mathrm{T}$ or $\mathrm{C}$. The nucleotides at the $3^{\prime}$ end are then exchanged with labeled dNTPs by the action of the Klenow fragment of DNA polymerase I, and the labeled product is cleaved to produce blunt ends (Figure 1).

Post-PCR labeling was originally developed for fluorescence-based singlestrand conformation polymorphism (SSCP) analysis. This labeling technique is obviously applicable to microsatellite genotyping and provides a solution to the problem of non-template nucleotide addition (5). Therefore, we investigated whether the post-PCR labeling technique offers high-quality microsatellite genotyping. We performed genotyping and linkage analysis of the dominant familial exudative vitreoretinopathy (FEVR) gene, which has been mapped to 11q13-23 $(8,10)$. Using clinical samples, the accuracy of allele identification by the post-PCR fluorescence-labeling technique was assessed. 


\section{MATERIALS AND METHODS}

\section{DNA and Markers}

Genomic DNA was extracted from 38 people from six families manifesting FEVR, the genetic locus for which has been assigned to $11 \mathrm{q} 13-23(8,10)$. A total of 45 dinucleotide markers was selected and flanked by the markers D11S987 and D11S35. The original primer sequences were obtained from the literature $(2,4)$ or the genome database at http://www.gdb.org and were modified to contain either ATT or GTT at their $5^{\prime}$ ends (6). Two markers were excluded from this study: D11S4196 contained an unspecified nucleotide in the original primer sequence data, and D11S4128 yielded poorly amplified target DNA and abundant extra products, despite the optimization of PCR. Therefore, 43 markers were used in this study.

\section{PCR}

PCR was carried out in a $5-\mu \mathrm{L}$ reaction mixture containing $1 \mu \mathrm{M}$ each primer, $0.2 \mathrm{mM}$ each of four nucleotides, 50 ng template DNA, 0.125 U Taq DNA polymerase (PE Biosystems, Foster City, CA, USA), 27.5 ng Taq-Start ${ }^{\mathrm{TM}}$ antibody (Clontech Laboratories, Palo Alto, CA, USA), $2.5 \mathrm{mM} \mathrm{MgCl}_{2}, 10$ $\mathrm{mM}$ Tris- $\mathrm{HCl}, \mathrm{pH} 8.3$, and $50 \mathrm{mM} \mathrm{KCl}$. The cycling profile was $1 \mathrm{~min}$ at $95^{\circ} \mathrm{C}$ for initial denaturation, 30 cycles of 30 $\mathrm{s}$ at $95^{\circ} \mathrm{C}$ for denaturation, $30 \mathrm{~s}$ at the optimized temperature for annealing and $60 \mathrm{~s}$ at $72^{\circ} \mathrm{C}$ for extension, followed by $5 \mathrm{~min}$ at $72^{\circ} \mathrm{C}$ for final extension. The optimal annealing temperature was determined for each marker using a Biometra ${ }^{\circledR}$ T GRADIENT thermal cycler (Biometra, Goettingen, Germany). One microliter of each PCR product was used for agarose gel electrophoresis to examine fragment size and yieldand to confirm the absence of nonspecific products.

\section{Multiplexing and Fluorescence Labeling}

Based on the agarose gel electrophoresis results, combinations of microsatellite markers for multiplex electrophoresis were chosen. Fluorescent dyes used in this study were $N, N^{\prime}-d i$ ethyl-2',7'-dimethyl-6-carboxyr hoda(R110), 6-carboxy-X-rhodamine (ROX) and $N, N, N^{\prime}, N^{\prime}$-tetramethyl-6-carboxyrhodamine (TAMRA). Fluorescently labeled deoxy-mononucleotides-R6GdCTP, R110-dUTP and TAMRAdCTP-were purchased from PE Biosystems. Labeling dyes were selected for each marker to ensure there would be no overlap of peaks of the same color in the marker profile. PCR products were mixed either before or after the labeling procedure. Before the procedure, an equal volume of each PCR product was pooled for each fluorescent dye. For the post-PCR labeling reaction, $4 \mu \mathrm{L}$ of the pool were mixed with $4 \mu \mathrm{L} 5 \mathrm{mM}$ Tris-HCl, $\mathrm{pH} 8.7,10$ $\mathrm{mM} \mathrm{MgCl}_{2}, 0.1 \mathrm{U} / \mu \mathrm{L}$ Klenow fragment DNA polymerase I and either 2 $\mu \mathrm{M}$ R6G-dCTP, $2 \mu \mathrm{M}$ R110-dUTP or 8 $\mu \mathrm{M}$ TAMRA-dCTP. The mixture was incubated for $15 \mathrm{~min}$ at $37^{\circ} \mathrm{C}$, and the reaction was stopped by adding $0.8 \mu \mathrm{L}$ $0.2 \mathrm{M}$ EDTA. Then, $0.8 \mu \mathrm{L}$ calf intestimine (R6G), 6-carboxyrhodamine

nal alkaline phosphatase (2 U) were added. The mixture was incubated for an additional $30 \mathrm{~min}$ at $37^{\circ} \mathrm{C}$ to degrade the labeled nucleotide. The samples that were separately labeled with the three fluorescent dyes were mixed into a single tube to make each marker set. After the labeling procedure, each PCR product $(4 \mu \mathrm{L})$ was independently labeled and then pooled in a single tube based on the marker sets described above. In both cases, seven marker sets were made, each consisting of 3-10 labeled products.

\section{Electrophoresis}

One microliter of the pooled sample was mixed with $0.5 \mu \mathrm{L}$ size standard (GS400HD [ROX]; PE Biosystems) and $14 \mu \mathrm{L}$ formamide, followed by heat denaturation for $3 \mathrm{~min}$ at $95^{\circ} \mathrm{C}$. Capillary electrophoresis was performed using an ABI PRISM ${ }^{\circledR} 310$ Genetic Analyzer (PE Biosystems) under the conditions for microsatellite analysis suggested by the manufacturer. Data

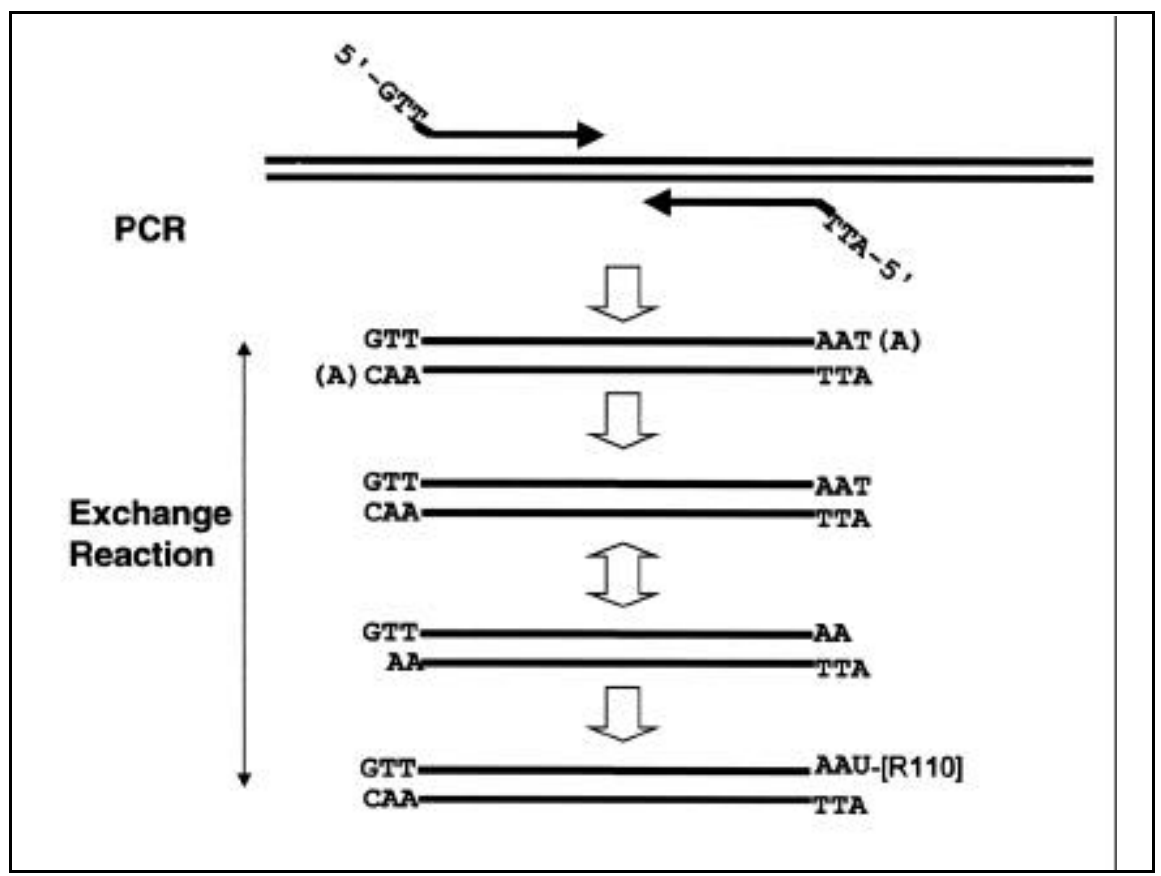

Figure 1. Principle of post-PCR fluorescence labeling. An example using R110-dUTP to replace the $3^{\prime}$-terminal T of a PCR product is shown. PCR is carried out using primers modified to contain ATT (or GTT) at their $5^{\prime}$ ends. R110-dUTP and Klenow fragment are added to the reaction tube to initiate a $3^{\prime}$ exchange reaction, which also removes any extra non-template nucleotide (A) added during the amplification reaction when Taq DNA polymerase is used. PCR product is efficiently labeled even in the presence of all four nucleotides, including TTP, because the DNA strand modified at its $3^{\prime}$ end with the fluorescent moiety is resistant to the $3^{\prime}$ exonuclease activity of the Klenow fragment. Consequently, the labeled product accumulates as is indicated by the unidirectional arrow in the figure. Either R110-, R6G- or TAMRA-labeled dUTP or dCTP can be used to replace the $3^{\prime}$-terminal $\mathrm{T}$ or $\mathrm{C}$, respectively. 
collection and allele identification were performed by GeneScan ${ }^{\circledR}$ and Genotyper $^{\mathrm{TM}}$ software (PE Biosystems). The size range and number of alleles were analyzed for each marker. Linkage analysis and haplotype construction were performed by GENEHUNTER 2 software (7).

\section{RESULTS}

Forty-three microsatellite markers in 38 subjects, a total of 1634 samples, were successfully analyzed by the postPCR fluorescence-labeling technique. The marker profiles, as shown in Figure 2 , revealed clear fragment peaks sepa- rated every 2 bp in all but two markers, which are discussed later. Fluorescent signal intensity was sufficient for the Genotyper allele identification system in almost all samples. Genotyping was accomplished by manually choosing either one (homozygous) or two (heterozygous) conclusive peaks of true alleles, which can be distinguished easily from stutters. Some markers (especially D11S4184, which yielded less PCR product) gave a weak fluorescent signal in the analysis of some subjects when the electrophoresis was multiplex. In such cases, genotyping was performed either by directly inspecting the profiles of the GeneScan results or by repeating the electrophoresis solely for that mark-

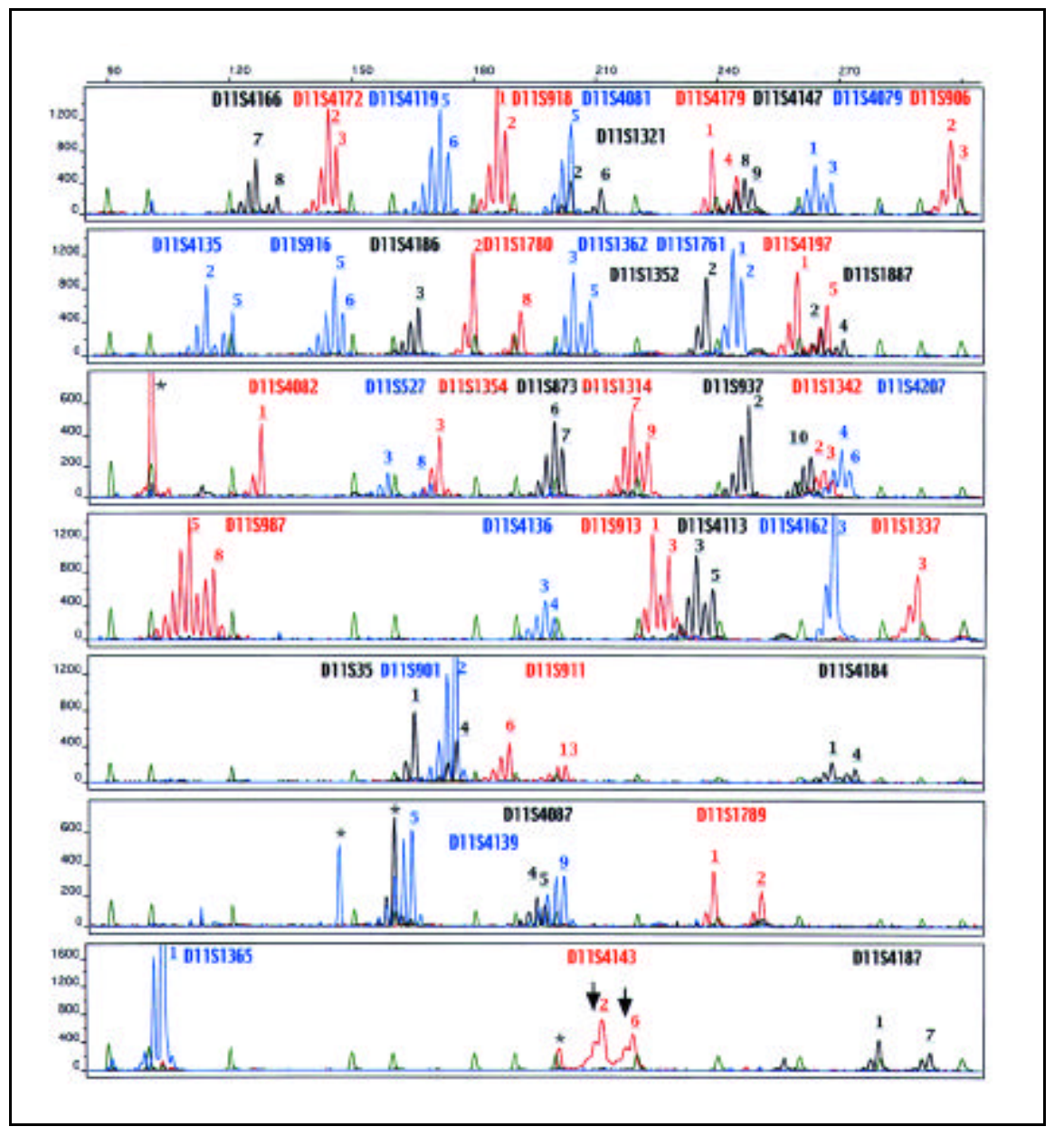

Figure 2. Multiplex microsatellite analysis of post-PCR fluorescently labeled markers. Names of the markers are shown above the allele peaks. Clearly separated peaks were observed for all markers except for D11S4143 (arrows). The extent of multiplexing is 10, 9, 8, 6, 4, 3 and 3, respectively, for each run from the top. The peaks colored in red, blue and black represent samples labeled with R6G-dCTP, R110-dUTP and TAMRA-dCTP, respectively. Peaks in green are those of size standards that are labeled with ROX. Alleles are numbered in ascending order on top of true peaks. Asterisks indicate fortuitous PCR products. The scale on the top bar indicates fragment size. Fluorescence intensity in arbitrary units is shown on the ordinate. er, followed by data confirmation with genotypes in the family. No ambiguous peaks attributable to the addition of a non-template nucleotide were detected.

Each marker contains 2-13 alleles (mean 7.2 alleles). The difference of the shortest and longest alleles in each marker ranged between 2 and $50 \mathrm{bp}$ (mean $15.7 \mathrm{bp}$ ). Although genotyping was possible for all markers, some caution was needed in genotyping four of them. Two markers showed poorly separated peaks for unknown reasons, either in all (D11S4143; Figure 2) or some (D11S4207) individuals. D11S1362 revealed peaks that were clearly separated by $2 \mathrm{bp}$, but the alleles in six members of one family could not be assigned because one stutter peak was unusually high. However, genotypes of these subjects could be estimated from those of the kinship. All markers showed results consistent with Mendelian inheritance, although interpretation of inheritance of D11S4119 in three families required the assumption of an allele that does not produce PCR product.

Multipoint linkage analysis of FEVR using genotypes established here showed evidence for linkage of this disease with the markers in all six families. Haplotyping revealed five centromeric and two telomeric recombinations that were informative in identifying the FEVR locus as the critical region between markers D11S4143 and D11S901, spanning $0.8 \mathrm{cM}$ (manuscript in preparation).

\section{DISCUSSION}

In this study, we have demonstrated that the products of post-PCR labeling are completely free from the addition of non-template nucleotides, which results in clean fragment peak separation and accurate genotyping. Analysis with GENEHUNTER software (7), which checks inheritance discrepancy, revealed that the genotypes established here are perfectly concordant with the family structure. Although a few markers showed anomalous features, including inefficient PCR amplification and the appearance of ambiguous stutter peaks, correct genotypes can be estimated by careful examination of the data. Thus, we feel this technique is 


\section{Research Report}

efficient for highly accurate genotyping.

The number of markers in multiplexed sets shown here is somewhat fewer than that in commercially distributed marker sets. This is because establishing maximum multiplexing of marker sets was not our goal and does not imply a limitation in our method. Multiplexing in conventional multimarker genotype analysis is cost effective and labor saving. This is also true for the post-PCR labeling approach described here. Three alternative temporal strategies are possible with mixing: $(i)$ mixing before PCR (multiplex PCR), (ii) mixing after PCR but before labeling and (iii) mixing after labeling. The necessary amount of fluorescent nucleotide is greatly reduced by strategies (i) and (ii). We tried multiplex PCR of five primer sets and succeeded with 41 (93\%) of the 43 markers (data not shown), although signals of some markers were not strong enough for straight genotyping. Further optimization should yield even greater signal intensity and easier interpretation of the data derived from multiplex PCR. Am biguity of amplification in multiplex PCR can be avoided by the use of the second mixing strategy. However, occasionally, the signal intensity of some markers may not be sufficient for multiplex analysis. Therefore, prior analysis of individual markers, together with knowledge of their size range and signal intensity, is strongly recommended in the design of multiplexing markers.

Multiplex microsatellite genotyping is usually done using fluorescently labeled primers that are typically obtained as sets of primers (11). Commercially available primer sets (e.g., Linkage Mapping Sets; PE Biosystems) contain primers that can be used in up to 300 reactions, and the cost is approximately $\$ 0.30 /$ marker/reaction, if all primers in the sets are exhaustively used. In the present post-labeling method, the cost for the reagents in the labeling reaction, including fluorescent nucleotides, Klenow fragment and alkaline phosphatase, is less than $\$ 0.20 /$ marker/reaction, if four markers are simultaneously labeled in one tube (quadruple labeling). The cost of primer pair synthesis without modification is approximately $\$ 0.10$ if they are used 300 times. Thus, for small- to medium-scale genotyping projects in which exhaustive use of all markers in the commercially available sets is unlikely, the present method of post-labeling is clearly more cost effective compared to using fluorescently labeled primers.

Moreover, the fluorescent dyes used for labeling in this method can be chosen at any stage after PCR so that the design of a set of markers for multiplexing is flexible. This is an important feature of the present method, especially when new markers are needed for inclusion in the typing experiments.

The disadvantage of post-PCR labeling is the requirement for additional processes-the Klenow fragment exchange reaction and phosphatase treatment. However, these processes are carried out in the same tube simply by successive additions of the reagents so that the whole process can be streamlined without significant additional cost. Incomplete phosphatase treatment potentially results in extra peaks in the marker profile that impede analysis. However, the phosphatase procedure is reliable, and problems rarely arise for experienced researchers. In conclusion, the post-PCR fluorescence-labeling technique is a valuable adjunct to genetic analysis as a precise method for microsatellite genotyping.

\section{ACKNOWLEDGMENTS}

This work was supported by Grantsin-Aid for Scientific Research on Priority Areas (Genome) from the Ministry of Education, Science, and Culture of Japan, and the Special Coordination Funds for Promoting Science and Technology from the Science and Technology Agency, Japan.

\section{REFERENCES}

1.Clark, J.M. 1988. Novel non-templated nucleotide addition reactions catalyzed by procaryotic and eucaryotic DNA polymerase. $\mathrm{Nu}$ cleic Acids Res. 16:9677-9686.

2.Dib, C., S. Fauré, C. Fizames, D. Samson, N. Drouot, A. Vignal, P. Millasseau, S. Marc et al. 1996. A comprehensive genetic map of the human genome based on 5,264 microsatellites. Nature 380:152-154.

3.Ginot, F., I. Bordelais, S. Nguyen and G. Gyapay. 1996. Correction of some genotyping errors in automated fluorescent microsatellite analysis by enzymatic removal of one base overhangs. Nucleic Acids Res. 24:540-541.

4.Gyapay, G., J. Morissette, A. Nignal, C. Dib, C. Fizames, P. Millasseau, S. Marc, G. Bernardi et al. 1994. The 1993-94 Généthon human genetic linkage map. Nat. Genet. 7:246-249.

5.Inazuka, M., T. Tahira and K. Hayashi. 1996. One-tube post-PCR fluorescent labeling of DNA fragments. Genome Res. 6:551-557.

6.Inazuka, M., H.-M. Wenz, M. Sakabe, T. Tahira and K. Hayashi. 1997. A streamlined mutation detection system: multicolor postPCR fluorescence labeling and single-strand conformational polymorphism analysis by capillary electrophoresis. Genome Res. 7:1094-1103.

7.Kruglyak, L., M.J. Daly, M.P. Reeve-Daly and E.S. Lander. 1996. Parametric and nonparametric linkage analysis: a unified multipoint approach. Am. J. Hum. Genet. 58:13471363.

8.Li, Y., B. Muller, C. Fuhrmann, C.E. van Nouhuys, H. Laqua, P. Humphries, E. Schwinger and A. Gal. 1992. The autosomal dominant familial exudative vitreoretinopathy locus maps on 11q and is closely linked to D11S533. Am. J. Hum. Genet. 51:749-754.

9.Mugnuson, V.L., S.J. Nylund, J.B. Rayman, J.I. Knapp, A.L. Lowe, S. Ghosh and F.S. Collins. 1996. Substrate nucleotide-determined non-templated addition of adenine by Taq DNA polymerase: implications for PCRbased genotyping and cloning. BioTechniques 21:700-709.

10.Müller, B., U. Orth, C.E. van Nouhuys, C. Duvigneau, C. Fuhrmann, E. Schwinger, H. Laqua and A. Gal. 1994. Mapping of the autosomal dominant exudative vitreoretinopathy locus (EVR1) by multipoint linkage analysis in four families. Genomics 20:317-319.

11.Pritchard, L.E., Y. Kawaguchi, P.W. Reed, J.B. Copeman, J.L. Davies, A.H. Barnett, S.C. Bain and J.A. Todd. 1995. Analysis of the CD3 gene region and type 1 diabetes: application of fluorescence-based technology to linkage disequilibrium mapping. Hum. Mol. Genet. 4:197-202.

12.Schwengel, D.A., A.E. Jedlicka, E.J. Nanthakumar, J.L. Weber and R.C. Levitt. 1994. Comparison of fluorescence-based semi-automated genotyping of multiple microsatellite loci with autoradiographic techniques. Genomics 22:46-54.

13.Wenz, H.-M., J.M. Robertson, S. Menchen, F. Oaks, D.M. Demorest, D. Scheibler, B.B. Rosenblum, C. Wike et al. 1998. High-precision genotyping by denaturing capillary electrophoresis. Genome Res. 8:69-80.

Received 17 March 2000; accepted 13 June 2000.

Address correspondence to:

Dr. Kenshi Hayashi

Kyushu University

Maidashi 3-1-1

Higashiku

Fukuoka, 812-8582, Japan

e-mail: khayashi@gen.kyushu-u.ac.jp 